\title{
SYPHILIS OF THE HEART AND AORTA, MORE ESPECIALLY THE EARLY SIGNS AND SYMPTOMS
}

By FREDERICK W. PRICE, M.D., F.R.S. (Edin.)

Based upon an Address delivered before the Medical Soziety for the

Study of Venereal Diseases, February 26th, I926.

To an audience such as this, it is, I feel sure, unnecessary to emphasise the great importance of the subject of our discussion this evening. No doubt most, if not all, of you are personally sadly acquainted with tragic cases of this affection. The subject is also one of great difficulty, more especially with regard to the relative frequency and diagnosis of the condition. These are intimately bound up with ætiological and pathological considerations.

I shall confine my remarks to the acquired form of the disease, for the reason that the congenital form lies almost entirely outside my experience.

Pathology and Relative Frequency.-It may be advisable if, first of all, I reminded you of the lesions which acquired syphilis may produce. The disease may attack the aorta, the cardiac valves, the coronary arteries, the myocardium, the auriculo-ventricular junctional tissues, and the pericardium. With regard to the aorta, Professor $H$. M. Turnbull found that in 288 necropsies at the London Hospital in which lesions of acquired syphilis were present, the most common syphilitic lesion was mesaortitis. This affection is the result of inflammation of the vasavasorum and their terminations in the external and middle coats of the aorta. There is perivascular infiltration of lymphocytes and plasma cells, accompanied by periarteritis and endarteritis-indeed, in the great majority of cases all the coats are involved. Ultimately the lumen of the vessels may become completely closed. The adventitia and, later, the media of the areas of the aorta supplied by the inflamed vasa-vasorum are secondarily affected, and the inflammation is mainly confined to these coats. The inflammation in the media is followed by absorption and, it may be, necrosis of the elastic 


\section{SYPHILIS OF THE HEART AND AORTA}

tissue and muscle-fibres, and, at a later period, by new formation of fibrous tissue. This leads to local weakening of the vessel, which may give rise to aneurysms, varying from a minute to large size. The intima over the areas of inflammation is usually thickened. The thickening of the intima is merely secondary, and is protective or compensatory. It is due to the connective tissue laminæ being increased in size and number, often an ingrowth of new vessel formation from the media, and sometimes cellular infiltration from the other coats of the aorta. In the early stages there is an absence of evidence of fatty and calcareous degeneration - a fact which may be due to the ingrowth of new vessels from the media. The secondary thickening of the intima has been confused with atheroma. Mott, Marchand, Turnbull and others have described the distinctive features of syphilitic mesaortitis and atheroma. It is necessary to point out and emphasise that this distinction is even yet not nearly sufficiently appreciated, and is not even admitted by tome. Professor Turnbull has shown that the secondary shickening of the intima in syphilitic mesaortitis may be distinguished from atheroma by the naked eye-by its greater latitude and prominence, its crenated outline, pearly colour, pitted surface, and rubber-like consistency. The pitting and scarring are due to the replacement of the media by fibrous tissue. Having pointed out these distinctive features, however, I hasten to add that in the later stages atheroma usually occurs in the thickened intima over the areas of inflammation; also that syphilitic mesaortitis and primary atheroma may co-exist, especially in later life.

The ascending part of the aorta is the most commonly affected. The disease-process in the aorta may involve the aortic valve, and occasionally it extends to the base of the aortic cusp of the mitral valve. The inflammation is similar to that of the aorta. In the case of the aortic valve, it results in incompetence and occasionally in stenosis. Syphilitic mesaortitis may also give rise to narrowing or complete occlusion of the orifice of one or both of the coronary arteries. Syphilis may also affect the terminal branches of the coronary arteries. In syphilis of the coronary arteries the aorta is usually affected at the same time. If, from any cause, the orifice, or the trunk, or one of the main branches of a coronary artery, be completely and 


\section{BRITISH JOURNAL OF VENEREAL DISEASES}

abruptly closed, sudden death, without the occurrence of structural changes, may occur ; or infarction and, later, secondary fibrosis may ensue, resulting in weakening of the cardiac wall. In those cases in which, from any cause, closure of a coronary artery or one of its main branches is gradual, or is only partial, and in cases of narrowing or occlusion of the terminal branches, infarction does not take place ; local anæmia results, and consequent atrophy of the muscle-cells and secondary fibrosis. Gummatous deposits, with secondary fibrosis, also occur in the cardiac wall, but they are of comparatively rare occurrence. It will thus be seen that in syphilis, fibrosis of the myocardium may be the result of coronary disease or gummata. Any of these lesions may involve the auriculo-ventricular junctional tissues and give rise to auriculo-ventricular heart-block. Syphilis of the pericardium is of rare occurrence. The disease more commonly attacks in the form of an infiltration, with consequent formation of fibrous tissue, resulting in adhesions between the two layers of the pericardium, while in some cases there is effusion of fluid into the pericardial sac. Gummata are of rare occurrence.

The foregoing is a brief summary of the lesions which it is generally agreed syphilis may produce. But this does not by any means exhaust this part of our subject. I am well aware that we are now entering upon a field of great difficulty and even of controversy; for, among other things, the whole subject of the pathology and ætiology of arterial disease is involved. But certain questions are of the greatest practical importance with regard to the relative frequency of syphilis as a cause of diseases of the heart and aorta, and differential diagnosis and treatment. Thus, in addition to the fact that syphilitic aortitis may involve the aortic valve and the base of the aortic cusp of the mitral valve, is syphilis among the causes of primary chronic endocarditis of one or more of the cardiac valves? There is no evidence that it is. Again, apart from inducing secondary fibrosis in the manner described, may syphilis give rise to fibrosis of the myocardium independently ?in other words, is it among the causes of primary chronic myocarditis; i.e., a true inflammation ? There is no doubt that syphilis may give rise to a primary chronic inflammation elsewhere; and it would appear that congenital syphilis does so in the case of the myocardium. Some 


\section{SYPHILIS OF THE HEART AND AORTA}

authorities are of opinion that acquired syphilis may cause a patchy or a diffuse fine fibrosis independently of the coronary arteries, while others are not. In any case, the histological diagnosis is very difficult even in the early stage, and is perhaps impossible in the later stage, so that it is probable that many of the cases of fibrosis of the myocardium in elderly people which are thought to be the result of syphilis are not so. Or again, and perhaps most important of all, is syphilis a cause of primary atheroma? In my opinion, it is not.

Syphilis is the cause of a large proportion of all cases of aortic incompetence, and also an important ætiological factor of angina, especially under the age of forty. But I would submit that, in considering the relative frequency of syphilis in affections of the heart and aorta, there is now a danger of going to the other extreme. Thus, statements to the effect that syphilis is responsible for from a quarter to a third of the total number of cases of organic disease of the heart, for about three-quarters of all cases of aortic regurgitation, and almost invariably for cases of aortic incompetence in which there is an absence of a definite history of acute rheumatism, should be accepted with the greatest reserve. In considering the relative frequency of syphilis as a cause of diseases of the heart and aorta, we have to consider the questions of a history of syphilitic infection, of a positive Wassermann reaction, and of other syphilitic stigmata during life ; and of the specific nature of, or the presence of, the Spironema pallidum in, the lesions of the heart or aorta themselves, or the presence of other undoubtedly syphilitic lesions, or of a positive Wassermann, on post-mortem examination. Of course syphilis of the heart and aorta would be of very frequent occurrence if all the lesions found in the heart and aorta post-mortem in those in whom there was a history of antecedent syphilitic infection, or of a positive Wassermann, or even of other syphilitic stigmata, during life, or even of a positive Wassermann after death, are to be regarded as syphilitic in origin. It is, I hope, not necessary to point out that such lesions are not necessarily syphilitic. Other evidence is required in order to be certain that a lesion is of specific origin. Such are : (I) the detection of the Spironema pailiaum in the lesion itself, or (2) the specific histological character of the lesion. 


\section{BRITISH JOURNAL OF VENEREAL DISEASES}

Symptoms and Diagnosis.-In aortitis pain is the most frequent and characteristic symptom, and it is exceedingly important to recognise it. It is situated behind the upper part of the sternum, and is generally localised there, but it may radiate to the arms and neck. It may vary from slight discomfort or a sensation of pressure to an intense pain. It may be more or less constant, or intermittent. Occasionally there are paroxysms of pain, accompanied by a feeling of suffocation. The pain may come on spontaneously-that is, apart from physical exertion, especially when lying down, or it may occur only on exertion; it is usually aggravated by exertion; it may not completely disappear on cessation of exertion. There may be hyperæsthesia over the painful area. Occasionally severe attacks of suffocation occur, and tachycardia may often be noted. The pain may disappear after a few months or weeks, either spontaneously or as the result of treatment. It may be followed by angina pectoris. Some observers are of opinion that sometimes, during the early stage, rubbing sounds along the right border of the sternum may be noted, especially when the patient leans forward ; personally I have never been able to detect this. At a later stage there may be indications of fusiform dilatation of the aorta. There may be pulsation in the neck, particularly in the supra-sternal notch, and in the first and second intercostal spaces on either side of the sternum; impairment of percussion over the manubrium sterni and on either side of it ; a systolic murmur over the aortic area; while the second sound may exhibit a musical, bell-like or clanging quality. With the X-rays the aorta may show diminished translucency and an increased diameter. Alteration in the voice and tracheal tugging are occasionally to be noted.

Later the clinical picture of aortic incompetence or of myocarditis or saccular aneurysm may develop. With regard to the first, shortness of breath on exertion is probably the most frequent symptom of all. The largest group present symptoms of systemic anæmia, such as giddiness, fainting attacks, early fatigue on mental effort, sleeplessness and headache. What is often termed "cardiac asthma" is comparatively frequent. Pain in the præcordium is of fairly frequent occurrence, but is not so common as in aortitis. Angina pectoris is relatively infrequent. Indications of chronic venous con- 


\section{SYPHILIS OF THE HEART AND AORTA}

gestion supervene in a comparatively small proportion of cases. General œdema is only occasionally met with. I would emphasise the great importance of an early and most careful search for the presence of a diastolic murmur over the base of the heart, over and on either side of the sternum, and at the apex-in fact, over the whole præcordial area. In the early stages the murmur is often faint, and may be audible only after emptying the chest after a deep inspiration, and may be heard on some occasions and not in others. There is less tendency for the systolic blood pressure to rise, and still more so for physical signs of left ventricular hypertrophy to develop, than in the case of aortic incompetence due to other causes; I have observed some cases which proceeded to a fatal termination with an absence of evidence of left ventricular hypertrophy during the whole course of the illness. Involvement of the myocardium may reveal itself on polygraphic and, more frequently, on electro-cardiographic examination, by the presence of the first or second grade of auriculo-ventricular block, and rarely by bundle-branch or intraventricular block, and in one case of my experience by transient attacks of auricular fibrillation. If complete auriculo-ventricular block occurs, it usually does so in the later stages. Subacute bacterial endocarditis may supervene. Sudden death may occur, and may be due to complete and abrupt closure of the orifice or trunk, or one of the main branches of a coronary artery, to rupture of the heart, fatal syncope or angina pectoris.

The foregoing description covers the great majority of cases. It is necessary to add the following : Relative, and even complete, recovery-either the result of specific treatment or spontaneous-may occur at any period. There may be exacerbations of the disease all though life. Sometimes aortitis escapes notice altogether, and the clinical picture is that of aortic incompetence developing insidiously. The clinical picture may be that of acute general cardiac failure, it may be proceeding to rapid dissolution. Sometimes the condition is only discovered post-mortem.

In the early stages the Wassermann reaction is almost invariably positive, but as the disease becomes more chronic it is increasingly negative.

The diagnosis is sometimes comparatively easy, but may be very difficult. In its consideration the following 


\section{BRITISH JOURNAL OF VENEREAL DISEASES}

points, among others, require consideration: The age, the previous history, the history of the present illness, the subjective symptoms, the results of physical and instrumental examination, the Wassermann reaction, the presence or otherwise of syphilitic stigmata, and the course of the disease. With regard to the previous history, may I remind you that it is very easy to overlook a rheumatic infection in childhood. There may also be a history of rheumatic and syphilitic, and even other, infection in the same patient. There may be a combined lesion. I would emphasise the importance of pain behind the upper part of the sternum, presenting the characteristic features described. If it has been possible to obtain a full and accurate history, it will be found that in the vast majority of cases it has been present in the early stages. The pain differs from that of angina pectoris in that it is less paroxysmal, its localisation is higher, it has a far less tendency to radiate to a distance, and it is more likely to come on spontaneously and not to cease completely after exertion is over. The presence of a systolic murmur over the aortic area, an altered second sound in the manner described, and the result of an examination with the $\mathrm{X}$-rays, are of considerable diagnostic importance. It is necessary to exclude aortitis due to infection by pyogenic organisms of a subacute or chronic type, by the tubercle bacillus, and other organisms, aneurysm and atheroma. The last-named, when limited to the aorta, however, rarely causes pain. A cardiac lesion coming on in middle life, if antecedent rheumatic infection can undoubtedly be excluded, is strongly suggestive of syphilis, as also is angina pectoris occurring before the age of forty. A combination of aortic and mitral valvular disease, especially before thirty years of age, is strongly suggestive of being of rheumatic origin. Atheroma much more frequently causes aortic stenosis than incompetence. A Wassermann test should be done in all doubtful heart cases, and in all cases of aortic disease, even if there is a history of acute rheumatism. I need not remind you that a negative Wassermann is not so valuable as a positive reaction; that a single negative reaction does not exclude syphilis; and that in some cases it may be advisable to employ a provocative dose of salvarsan or one of the other organic arsenical preparations. We should, on the other hand, be careful not to place too much reliance on the result of the 


\section{SYPHILIS OF THE HEART AND AORTA}

Wassermann reaction; it should be considered in conjunction with other clinical data. A positive Wassermann does not indicate that the malady from which the patient is suffering is necessarily syphilitic; the same applies to the co-existence of other specific stigmata. There may also be a double lesion.

Syphilis of the pericardium is usually latent. A diagnc sis is sometimes possible from the discovery of the clinical features of pericarditis in a known syphilitic subject.

Prognosis.-This depends largely, perhaps very largely, upon early diagnosis and the kind of treatment adopted. Taking cases as a whole, the prognosis is very unfavourable, due, no doubt, to the fact that a correct diagnosis is not often made until the later stages of the disease. I venture to hope that in future an early diagnosis will be arrived at with increasing frequency. When untreated, the lesion is usually progressive, it may be rapidly so, and the mortality is high. If, on the other hand, a diagnosis is made early, and treatment is prompt and adequate, especially when the patient has not received specific treatment previously, there is a reasonable prospect of relative recovery, and occasionally recovery is even complete. Naturally the prognosis is much more favourable in the absence of aortic incompetence, involvement of the coronary arteries and of the myocardium, angina pectoris and aneurysm Prompt. and adequate treatment of aortitis may prevent the aortic valve, coronary arteries and the myocardium becoming involved, and the formation of a saccular aneurysm. Even in the absence of angina pectoris and aneursym, in aortic regurgitation the prognos:s is usually very unfavourable, death often taking place within two or three years after the onset of subjective symptoms. Two important points in all cases are the degree of cardiac failure present and its rate of advance. Help is sometimes obtained from the results of polygraphic and, still more frequently, of electro-cardiographic examination. Thus the presence of alternation of the heart in the absence of a severe grade of tachycardia is an indication of extreme exhaustion of the heart muscle ; the occurrence of partial heart-block is a sign of myocardial involvement; persistent heart-block of mild degree is also indicative of myocardial damage ; bundle-branch block is usually of very unfavourable significance; and intraventricular block is 


\section{BRITISH JOURNAL OF VENEREAL DISEASES}

usually of grave import. A few cases of complete auriculoventricular block have been recorded in which energetic specific treatment appears to have been rewarded with recovery, but as far as my experience is concerned antispecific treatment is of no avail at this stage. Lastly, in all types of cases, marked and surprising improvement is occasionally met with when adequate specific treatment is adopted.

Treatment.-The various measures which may be applicable to any form of cardiac disorder should be considered in detail. Apart from these, the indication is for antisyphilitic treatment. This should be adopted in every case in which a definite diagnosis of syphilis of the heart or aorta is arrived at, even if the Wassermann reaction is negative; and in every case of disease of the heart or aorta in which there is a positive Wassermann or other manifestations of active syphilis, even in the absence of clinical evidence that the lesions of the heart and aorta are of specific origin. Cardiac failure is not a contraindication. It is impossible to exaggerate the importance of the adoption of this method of treatment promptly. It should also be prolonged. I am a firm believer in the employment of the arsenical preparations-but only under certain conditions. Their indiscriminate use is attended with danger, even of grave degree ; they may result in further swelling of the intima of the small vessels, and thus increase the risk of thrombosis. I always recommend a preliminary course, of three weeks' duration, of mercurial inunction and of iodide of potassium by the mouth-Io grains thrice daily during the first week, increased to I5 grains during the second week, and to 20 grains during the third. This to be followed by one of the arsenical compounds administered cautiously, commencing with small doses and only gradually increasing the dosage. The scheme I advise is the result of two or three conversations I have had the privilege of having with Colonel Harrison, to whom I desire gratefully to acknowledge my indebtedness. This consists of a series of courses of sulfarsenol injected subcutaneously, with mercury injected intramuscularly. The first course makes a cautious start with Io centigrammes of sulfarsenol, and the dosage is increased, in bi-weekly injections, to 24 centigrammes, the total amount given in the first fiftyfour days being 2.76 grammes in sixteen injections, with 


\section{SYPHILIS OF THE HEART AND AORTA}

8 grains of mercury in eight injections. The reason that the dosage of sulfarsenol in the first course is cautious is that patients with syphilis of the heart or aorta seem easily to be upset at first by arsenobenzol preparations. After the foregoing course of injections, potassium iodide is given for three weeks, to be followed three weeks later by a course of five injections of sulfarsenol and mercury. This time it may be possible to proceed more boldly with the sulfarsenol-three injections of 48 centigrammes and two of 60 centigrammes. Thereafter the treatment is continued with courses of ten injections of $5 \cdot 8$ grammes sulfarsenol and ro grains of mercury at intervals increasing from two to five months, with potassium iodide for a month at a time between the courses. The treatment is continued on these lines for two years. After the completion of this scheme, I suggest a three weeks' course of potassium iodide every two or three months during the rest of the patient's life. This method of treatment applies also to cases of aneurysm. It may be advisable to continue anti-syphilitic treatment as long as the Wassermann is positive even in the absence of other manifestations of active syphilis, for there is a risk of exacerbations all through life, and the small vessels around the principal lesions may become involved. I have been surprised at the trequency with which a positive Wassermann persists even in the case of patients who, from a clinical point of view, have done well.

I would urge the importance of further combined investigation, by the clinician and the pathologist, of this important subject.

In conclusion, I wish to express my thanks to Professor H. M. Turnbull * for his kindness in allowing me the opportunity of a most helpful conversation with him regarding the pathology of the subject.

* Turnbull, H. M.: The Quarterly Journal of Medicine, April, I9I5 (Vol. 8, No. 3I). 